\title{
Reminding and mood-congruent memory
}

\author{
STEPHEN G. GILLIGAN and GORDON H. BOWER \\ Stanford University, Stanford, California
}

\begin{abstract}
Earlier experiments found that happy or sad subjects learn more about prose materials that are affectively congruent with their feelings. This study asked whether this memory effect was mediated by selective reminding. Subjects induced through hypnosis to feel happy or sad indicated whether or not happy or sad prompting phrases reminded them of a personal experience; they also rated the phrase's imagery. Later, free recall showed the mood-congruity effect, which related strongly to imagery ratings but only weakly to remindings. In particular, the differential recall of happy versus sad phrases was about the same regardless of whether the phrases provoked reminiscences. Thus, selective reminding is at best a weak contributor to the moodcongruity effect in memory.
\end{abstract}

We are interested in how a person's emotional mood influences what he learns and remembers. In earlier work (Bower, Gilligan, \& Monteiro, 1981), we found a moodcongruity effect, in that people learned more about prose material that agreed with their mood during learning. In those experiments, subjects read narratives while feeling happy or sad and later recalled those narratives. Across experiments, the narratives differed: One story described a happy and a sad student meeting and playing a game of tennis; a second story described a patient recounting to his psychiatrist a series of happy and sad incidents from his life; a third described a happy and a sad character, each recounting a series of happy and sad incidents from his life. Later, free recall showed that happy readers learned more about happy material (character or incident) and sad readers learned more about sad material.

Several hypotheses to explain the mood-congruity effect have been advanced (Bower, 1981). One hypothesis, selective reminding, was suggested by subjects' introspections. This states that as subjects read about specific incidents in someone's life, they are reminded spontaneously of similar incidents in their lives. Moreover, such remindings are retrievals that are presumed to be mood-state dependent (see Bower, 1981): Specifically, happy-story incidents should be more likely to remind happy readers of (happy) autobiographical events, and sad-story incidents should be more likely to remind sad readers of (sad) autobiographical events. Finally, the hypothesis assumes that reminding is itself memory enhancing. If the story incident contacts an autobiographical episode, that episode can be used to enrich and elaborate upon the story incident, thus enhancing the latter's memory strength and recallability

This research was supported by a Research Grant MH-13950 to the second author from the National Institute of Mental Health. Requests for reprints may be mailed to Gordon Bower, Department of Psychology, Stanford University, Stanford, California 94305 . (see Anderson, 1976, for a statement of the elaboration theory). In agreement with this hypothesis, Bower and Gilligan (1979) found that subjects better remembered phrases that reminded them of an autobiographical event than they did phrases that did not provoke reminding experiences.

A second hypothesis to explain the mood-congruity effect is that, the more intense is the emotion experienced in conjunction with an event, the greater is the memory for that event (see Dutta \& Kanungo, 1975). We may suppose that happy story incidents elevate the intensity of happy readers' emotional states, but lower that of sad readers' states; conversely, a sad story incident has the reverse effect, lowering the mood intensity of the happy subject and elevating that of the sad subject. These are socially appropriate mood shifts. The hypothesis further assumes that material learned in association with more intense feelings is better remembered. This configuration of assumptions implies the better learning of mood-congruent material.

The experiment reported here investigated the selective-reminding hypothesis directly, while examining mood influences on selective learning. The procedure followed that used earlier by Bower and Gilligan (1979). In an incidental-learning task, subjects read many brief descriptive phrases (e.g., "a painful spanking") and had only a brief time within which to indicate whether each phrase reminded them of a specific autobiographical event. Half the phrases referred to happy incidents, and half referred to sad incidents. During this orienting task, half the subjects had been induced by a hypnotic suggestion to feel happy and half to feel sad. Later, while in a neutral mood, the subjects tried to recall the prompting phrases. We were interested in how the type of material and the subjects' mood influenced their reports of reminding experiences and their later recall.

\section{METHOD}

\section{Subjects}

The subjects were 16 adults who had just completed an 
8-week training course in self-hypnosis taught by the first author. All were well practiced in self-hypnosis and scored very high (10-12) on the Stanford Susceptibility Scale, Form C (see Weitzenhoffer \& Hilgard, 1967). They were well educated, either being graduate students or currently working as mentalhealth professionals.

\section{Materials}

The experimental materials were 20 happy and 20 sad phrases that described some possible incident, object, or state. All were of the form article-adjective-noun. Examples of happy phrases are "a euphoric moment," "a wet kiss," and "a comfortable chair." Examples of sad phrases are "a failed exam," "a grandparent's death," and "a painful spanking." The final phrases used were selected by a rating procedure from an initial pool of 60 . Five graduate students rated a pool of 30 happy and 30 sad phrases on a -7 to +7 happiness scale. The 20 happiest and 20 saddest phrases were selected for use.

These 40 phrases were ordered randomly in a booklet, 1 per page, with 3 irrelevant "primacy buffers" (phrases) placed at the front of the booklet and 3 irrelevant "recency buffers" at the end. On the page with each phrase was a place for subjects to answer (by circling Yes or No) whether they could access a specific personal memory using the phrase. The page also contained a 7-point scale on which the subject rated the vividness of any imagery evoked by the descriptive phrase. The scale was ordered from 7 ("intensely vivid image") down to 1 ("very vague"), and the subject circled a number from 1 to 7 .

\section{Procedure}

The subjects were tested in groups of three or four. The hypnotic mood-induction procedure was that used successfully in our previous work; for a fuller description, see Bower et al. (1981). The subjects were hypnotized rapidly with a modified eye-closure form of induction, with which all were familiar. Once in trance, the subjects were asked to remember a specific happy (or sad) event of their lives and to relive the emotion associated with that event. Suggestions were made for deepening the intensity of that mood: however, care was taken that the subjects would not be overwhelmed by the feeling (e.g., we prevented the sad subjects from crying). Next, the subjects were instructed to detach this emotion from the remembered event, to just experience the "pure emotion," and to maintain it as they read and rated the phrases in the booklet.

Once in the suggested happy or sad mood, the subjects were given the test booklet of 46 phrases and instructed regarding the ratings desired. Specifically, they were to use each phrase to try to access a specific personal experience and to indicate whether they had succeeded. They turned the pages of the booklet every $14 \mathrm{sec}$ on a cue ("Turn"). Nine seconds after the subjects had turned to a new phrase, the experimenter said "All right," indicating that the subjects had $5 \mathrm{sec}$ in which to circle Yes or No, reporting access of a memory of a personal experience by that phrase, and to circle a number from 1 to 7 , indicating the vividness of any image the phrase evoked, whether or not they had accessed a personal memory.

After completing the booklet, the subjects' mood was returned to neutral and they were taken out of trance. This required 10-12 $\mathrm{min}$. Then they performed a distracting task (adding and subtracting 4-digit numbers) for $5 \mathrm{~min}$. They then received a surprise free-recall test. Given a sheet of paper, they were asked to recall in writing all the descriptive (prompting) phrases they could remember from the booklet. They had as long as they desired to recall, with a required minimum of $5 \mathrm{~min}$; all were finished within $6 \mathrm{~min}$. Following the free-recall test, a cued-recall sheet was handed out. This listed on two sheets the 40 article-adjectives of the phrases with the nouns missing (e.g., "a euphoric __. "a grandparent's ___."The subjects had $20 \mathrm{~min}$ in which to fill out the cued-recall sheet. After normalization of their mood and having assured ourselves of no negative aftereffects, the subjects were debriefed and dismissed.

\section{RESULTS}

The protocols were scored for gist recall, with each phrase categorized as happy or sad. In free recall, most modifier-noun phrases were recalled as whole units, although half-credit was given for the noun or modifier alone. The free-recall percentages for happy readers were $39 \%$ for the happy phrases and $24 \%$ for the sad phrases. In contrast, recall percentages for the sad readers were $26 \%$ for the happy phrases and $28 \%$ for the sad phrases. An analysis of variance on these percentages found that happy phrases were remembered better overall than were sad phrases $[F(1,14)=16.6, p<.01]$, that happy readers did not recall significantly more than did sad readers, but that the affect of the phrase interacted in recall with the mood of the reader $[\mathrm{F}(1,14)=26.8$, $\mathrm{p}<.01$ ]. In particular, happy readers recalled happy phrases more than they did sad ones, whereas sad readers recalled sad phrases slightly more than they did happy ones. This interaction was as predicted by the moodcongruity hypothesis.

\section{Reminding Experiences}

Only 14 of the 16 subjects correctly indicated on the booklet whether each phrase reminded them of an event, object, or person in their lives. The other 2-1 from each mood group-were dropped in this analysis because they misunderstood the instructions calling for reminding reports and ratings. Therefore, the reminding and imagery results are based on 7 subjects per mood condition.

Our happy phrases proved to be very evocative: They produced reminding experiences $89 \%$ of the time for happy subjects and $92 \%$ for sad subjects, an insignificant difference (see Table 1). The sad phrases produced reminding experiences $84 \%$ of the time for sad subjects and $68 \%$ for happy subjects. The number of reminding experiences was analyzed statistically: Happy phrases produced more reminiscences than did sad ones overall $[F(1,12)=7.12, p<.05]$, sad and happy subjects did not differ significantly in total numbers of reminiscences, and the interaction between mood and phrase type was not statistically significant. A post hoc comparison of reminding rates by sad phrases for happy (69\%) versus

Table 1

Numbers of Reminding Successes and Failures and Their Conditional Free Recall for Happy and Sad Phrases by Happy and Sad Subjects

\begin{tabular}{lccccc}
\hline & \multicolumn{2}{c}{ Happy Mood } & & \multicolumn{2}{c}{ Sad Mood } \\
\cline { 2 - 3 } \cline { 5 - 6 } & $\mathrm{N}$ & PCR & $\mathrm{N}$ & PCR \\
\hline & & \multicolumn{3}{c}{ Happy } & Phrases \\
Reminded & 125 & .37 & 129 & .27 \\
Not & 15 & .36 & 11 & .13 \\
& \multicolumn{5}{c}{ Sad Phrases } \\
Reminded & 96 & .22 & 118 & .28 \\
Not & 44 & .20 & 22 & .25 \\
\hline
\end{tabular}

Note $-N=$ number of cases; $P C R=$ percent conditional recall. 
sad subjects $(84 \%)$ found them to differ significantly $(\mathrm{z}=3.15, \mathrm{p}<.01)$.

Next, the recall probabilities were calculated for phrases for which reminiscence succeeded and were contrasted with recall for phrases for which reminiscence failed (see Table 1). The low frequency of reminiscence failures in most conditions prevented a complete analysis contrasting recall rates for happy versus sad phrases that succeeded or failed to remind the subjects of a personal experience. For happy subjects, weighted recall rate was $31 \%$ for phrases that evoked reminiscence and $25 \%$ for phrases that failed for a given individual. For sad subjects, weighted recall rate was $28 \%$ for phrases that evoked reminiscence and $21 \%$ for phrases that failed. An analysis of variance on arcsin transforms of these conditional recall rates found no main effect of mood group but a marginally significant effect contrasting recall of phrases that succeeded versus failed in evoking reminiscences $[F(1,12)=4.05, p<.10]$. We conclude that there was only a small recall advantage for phrases that earlier had produced a reminding experience.

\section{Imagery Ratings}

The subjects rated each descriptive phrase on a 7 point scale for the imagery it evoked, regardless of whether it reminded them of a personal experience. For happy subjects, happy phrases evoked more vivid imagery (5.08) than did sad phrases (3.33). For sad subjects, happy phrases also evoked higher imagery ratings (5.30) than did sad phrases (4.76). An analysis of variance found that happy phrases evoked overall more vivid imagery than did sad phrases $[\mathrm{F}(1,12)=8.82$, $p<.05]$, but the main effect of the subject's mood was insignificant, as was the interaction between mood and type of phrase.

Phrases that succeeded in reminding subjects of a personal experience were rated as evocative of more vivid imagery (5.12) than were phrases that failed to produce reminiscence (1.89). There were too few reminiscence failures to perform analyses on imagery of happy or sad phrases that succeeded or failed in reminiscence.

Imagery rating for a phrase correlated with its recall. For both mood groups and both phrase types, recalled phrases had received higher imagery ratings (averaging 5.14) than had nonrecalled phrases (4.17), a significant difference $(\mathrm{p}<.01)$.

To eliminate subject differences in average scale range, another analysis divided each subject's imagery ratings into his or her higher and lower halves, and recall of phrases in each half was examined. Free-recall probabilities were .34 for high- and .23 for low-imagery phrases $[t(13)=3.74, p<.01]$. Cued-recall probabilities were .43 for high- and .37 for low-imagery phrases $[\mathrm{t}(13)=2.16, \mathrm{p}<.05]$. This correlation of imagery with recall might explain the better memory for happy phrases, since they evoked more vivid imagery than did sad phrases.

\section{Cued Recall}

The cued-recall test used the adjective modifier as a cue for recall of the last noun of the descriptive phrase. Such cues prompted the happy subjects to correctly recall $49 \%$ of their happy phrases and $41 \%$ of their sad phrases. Cued recall for the sad subjects was $38 \%$ for happy and $33 \%$ of sad phrases. Thus, the cued-recall probabilities of happy and sad phrases for the sad subjects were ordered oppositely to the ordering of their free-recall probabilities. An analysis of variance on these cued-recall percentages found that happy phrases were recalled better overall $[F(1,14)=5.25, p<.05]$, but the difference between mood groups was insignificant $[F(1,14)=2.70, p>.10]$, as was the interaction of mood group with type of phrase. Thus, the moodcongruity effect did not appear in cued recall as it had in free recall.

\section{DISCUSSION}

This experiment has yielded a somewhat complex pattern of results. The mood-congruity interaction was obtained as predicted in free recall, but not in cued recall. The pattern of results is similar to that obtained with state-dependent retrieval, namely, state dependency with free recall but not with adequately cued recall (see Eich, 1980). Possibly the mood-congruity effect resides in the strength of the association from the list-context to the memory node encoding each adjective-norm phrase (which list association influences free recall), whereas the association among elements of the phrase is already strong and depends largely on preexperimental factors, not mood congruity (so cued recall is unaffected). Interpreting the higher cued recall of happy versus sad phrases is problematic until we check whether the associative potency of the adjective cue for the noun is equated for our two sets of phrases.

Incidentally, the absence of a mood-dependent effect on cued recall here suggests that our free-recall results are not due just to subjects' complying with subtle "demand expectations" of the hypnotist-experimenter. The demand hypothesis would seem to predict that, the greater the clues for the "appropriate" response, subjects would perform more in conformity with mood-dependency expectations. Thus, this predicts, contrary to the data, that mood dependency should be more in evidence when the cues for recall are conspicuously pleasant or unpleasant adjectives rather than undifferentiated "list" cues. This is another example of the demand hypothesis's not accounting for details of memory data on mood congruity and mood state dependency.

The selective-reminding hypothesis was only weakly supported. Although sad subjects were reminded more often by sad phrases than were happy subjects, the two mood groups did not differ in reminiscences evoked by happy phrases. Perhaps our happy phrases were just too evocative to provide a sensitive baseline for assessing mood effects. Nonetheless, although phrases that produced reminiscences were somewhat better recalled than those that did not, such reminiscences did not seem to explain the mood-congruity interaction in recall of happy versus sad phrases by subjects feeling happy or sad during learning.

Our results may be related to those reported by Teasdale and Fogarty (1979). They examined the time happy or sad subjects required to retrieve a stipulated pleasant or unpleasant memory to a neutral prompting word (e.g., city, train). Subjects who had been made sad by the Velten (1968) procedure took longer to retrieve a pleasant memory than did subjects who had been made happy by Velten's procedure; but the two mood groups did not differ in latency to retrieve an unpleasant memory. The results 
of Teasdale and Fogarty paralleled those of Lloyd and Lishman (1975), who found that the time required to retrieve an unpleasant memory relative to that required to retrieve a pleasant memory to a prompting word correlated positively with subjects' degree of clinical depression. Our experiment differed from those earlier ones in that we used affective phrases (denoting possible events) rather than neutral nouns, and we did not stipulate that a happy or a sad memory should be retrieved. Our reminiscence measure was the proportion of subjects who reported being reminded of some specific experience within $14 \mathrm{sec}$, whereas the earlier investigators had measured retrieval latency. Perhaps we would have obtained an interaction in reminiscence between mood and phrase type had we measured retrieval latency. In any event, the observed success or failure of our subjects to be reminded by happy or sad phrases could not explain their later mood-congruent free recall.

A strong predictor of recall was the imagery evoked by the prompting phrase. Twelve of 14 subjects rated phrases that they recalled as being more imageable than phrases that they failed to recall. Another correlate of imagery rating was the success or failure of the phrase to evoke reminiscence of a personal experience. Also, the imagery evoked by a phrase depended on the subject's mood: Happy phrases evoked much more vivid imagery than did sad phrases for happy subjects, but not for sad subjects. This interaction in imagery parallels the mood-congruity interaction in free recall; that is, compared with sad subjects, happy ones showed a greater difference in recall of pleasant versus unpleasant phrases.

A simple description of the findings is that our happy phrases evoked more reminiscences, more imagery, and more cued recall from all subjects regardless of their mood. The only deviation from this pattern was a mood-congruity effect in free recall, in which sad subjects recalled slightly more sad than happy phrases. Our earlier work (see Bower et al., 1981) has always used free (rather than cued) recall to examine the mood-congruity effect. The free-recall task would seem to be more appropriate for showing mood-context effects, since free recall requires subjects to reinstate the contextual cues of acquisition, including their mood and mental set at the time. In reinstating the mood, the subject gains access selectively to those event memories most primed or activated by the mood. In contrast, a test for strongly cued recall (or recognition memory) will retrieve the stored event relatively directly, bypassing the need for a moodaided search of memory. An associative-network model to ex- plain the recall versus recognition difference in mood dependency was presented by Bower (1981). The explanation is similar to that given by Eich (1980) for studies showing drug-statedependent retrieval using free recall but not recognition measures of memory.

In conclusion, our main point is that the mood-congruity effect on free recall was replicated, but it did not appear to be mediated by mood-selective reminding during learning. For both mood groups, recall of happy versus sad phrases showed about the same difference when the phrases provoked a reminiscence as when they did not. Thus, one cannot use differential remindings to explain the differential recall of pleasant versus unpleasant materials by happy and sad subjects.

\section{REFERENCES}

Anderson, J. R. Language, memory and thought. Hillsdale, N.J: Erlbaum, 1976.

Bower, G. H. Mood and memory. American Psychologist, 1981, 36, 129-148.

Bower, G. H., \& Gilligan, S. Remembering information related to one's self. Journal of Research in Personality, 1979, 13, 420-432.

Bower, G. H., Gilligan, S. G., \& Monteiro, K. P. Selectivity of learning caused by affective states. Journal of Experimental Psychology: General, 1981, 110, 451-473.

Dutta, S., \& Kanungo, R. N. Affect and memory: $A$ reformulation. Oxford, England: Pergamon Press, 1975.

Eich, J. E. The cue-dependent nature of state-dependent retrieval. Memory \& Cognition, 1980, 8, 157-173.

Lloyd, G. G., \& Lishman, W. A. Effect of depression on the speed of recall of pleasant and unpleasant experiences. Psychological Medicine, 1975, 5, 173-180.

Teasdale, J. D., \& Fogarty, F. J. Differential effects of induced mood on retrieval of pleasant and unpleasant events from episodic memory. Journal of Abnormal Psychology, 1979, 88, 248-257.

Velten, E. A laboratory task for induction of mood states. Behaviour Research and Therapy, 1968, 6, 473-482.

Weitzenhoffer, A. M., \& Hilgard, E. R. Revised Stanford Profile Scales of Hypnotic Susceptibility Forms I, II. Palo Alto, Calif: Consulting Psychologist Press, 1967.

(Manuscript received for publication August 29, 1983.) 